\title{
Variational iteration method for fractional calculus - a universal approach by Laplace transform
}

\author{
Guo-Cheng $\mathrm{Wu}^{1,2^{*}}$ and Dumitru Baleanu $\mathrm{u}^{3,4,5^{*}}$
}

\author{
*Correspondence: \\ wuguocheng2002@yahoo.com.cn; \\ dumitru@cankaya.edu.tr \\ ${ }^{1}$ College of Mathematics and \\ Information Science, Neijiang \\ Normal University, Neijiang, 641112, \\ China \\ ${ }^{3}$ Department of Mathematics and \\ Computer Sciences, Cankaya \\ University, Balgat, Ankara 06530, \\ Turkey \\ Full list of author information is \\ available at the end of the article
}

\begin{abstract}
A novel modification of the variational iteration method $(\mathrm{VIM})$ is proposed by means of the Laplace transform. Then the method is successfully extended to fractional differential equations. Several linear fractional differential equations are analytically solved as examples and the methodology is demonstrated.
\end{abstract}

MSC: 39A08; 65K10; 34A12

Keywords: variational iteration method; fractional calculus; Laplace transform; symbolic computation

\section{Introduction}

The Lagrange multiplier technique [1] was widely used to solve a number of nonlinear problems which arise in mathematical physics and other related areas, and it was developed into a powerful analytical method, i.e., the variational iteration method $[2,3]$ for solving differential equations. The method has been applied to initial boundary value problems [4-9], fractal initial value problems [10, 11], $q$-difference equations [12] and fuzzy equations [13-15], etc.

Generally, in applications of VIM to initial value problems of differential equations, one usually follows the following three steps: (a) establishing the correction functional; (b) identifying the Lagrange multipliers; (c) determining the initial iteration. The step (b) is very crucial. Applications of the method to fractional differential equations (FDEs) mainly and directly used the Lagrange multipliers in ordinary differential equations (ODEs) which resulted in poor convergences. This point of view needs some explanations will elucidate the target of the suggested improvement, among them:

(1) When the Riemann-Liouville (RL) integral emerges in the constructed correctional functional, the integration by parts is difficult to apply;

(2) To avoid this problem, the RL integral is replaced by an integer one which allows the integration by parts. This is a very strong simplification but it affects the next steps of the application of the method;

(3) Therefore, the Lagrange multiplier is determined by a simplification not reasonably explained in the literature, so far.

To overcome this drawback, the present article conceives a method how the Lagrange multiplier has to be defined from Laplace transform. The technique can be readily and

(c) 2013 Wu and Baleanu; licensee Springer. This is an Open Access article distributed under the terms of the Creative Commons Attribution License (http://creativecommons.org/licenses/by/2.0), which permits unrestricted use, distribution, and reproduction in any medium, provided the original work is properly cited. 
universally extended to solve both differential equations and FDEs with initial value conditions.

\section{Basics of the variation iteration method}

In order to illustrate the basic idea of the technique, consider the following general nonlinear system:

$$
\frac{d^{m} u}{d t^{m}}+R[u]+N[u]=g(t)
$$

where $u=u(t), R$ is a linear operator, $N$ is a nonlinear operator and $g(t)$ is a given continuous function and $d^{m} u / d t^{m}$ is the term of the highest-order derivative.

The basic character of the method is to construct the following correction functional for Eq. (1):

$$
u_{n+1}=u_{n}+\int_{0}^{t} \lambda(t, \tau)\left(\frac{d^{m} u}{d \tau^{m}}+R\left[u_{n}\right]+N\left[u_{n}\right]-g(\tau)\right) d \tau,
$$

where $\lambda(t, \tau)$ is called the general Lagrange multiplier [1-3] and $u_{n}$ is the $n$th order approximate solution.

According to the VIM's rules $[2,3,16]$, readers may note that the integration by parts plays an important role in the derivation of the Lagrange multipliers. But in fractional calculus, generally, the following integration by parts cannot hold (as it is mentioned in point (2) of the preceding section):

$$
{ }_{0} I_{t}^{\alpha} v_{0}^{C} D_{t}^{\alpha} u=\left.[u v]\right|_{0} ^{t}-{ }_{0} I_{t}^{\alpha} u_{0}^{C} D_{t}^{\alpha} v,
$$

where $v=v(t),{ }_{0}^{C} D_{t}^{\alpha}$ and ${ }_{0} I_{t}^{\alpha}$ are the notations of the Caputo derivative and the RL integration, respectively. That's why the VIM was not so successful as other analytical methods such as the Adomian decomposition method (ADM) [17-19] and the homotopy perturbation method (HPM) [20-22] in fractional calculus. For this reason, we consider the following reconstruction of the method using the Laplace transform.

\section{New identification of the Lagrange multipliers}

Let us revisit the original idea of the Lagrange multipliers in the case of an algebraic equation. Firstly, an iteration formula for finding the solution of the algebraic equation $f(x)=0$ can be constructed as

$$
x_{n+1}=x_{n}+\lambda f\left(x_{n}\right) .
$$

The optimality condition for the extreme $\frac{\delta x_{n+1}}{\delta x_{n}}=0$ leads to

$$
\lambda=-\frac{1}{f^{\prime}\left(x_{n}\right)},
$$

where $\delta$ is the classical variational operator. From (4) and (5), for a given initial value $x_{0}$, we can find the approximate solution $x_{n+1}$ by the iterative scheme for (5)

$$
x_{n+1}=x_{n}-\frac{f\left(x_{n}\right)}{f^{\prime}\left(x_{n}\right)}, \quad f^{\prime}\left(x_{0}\right) \neq 0, n=0,1,2, \ldots .
$$


This algorithm is well known as the Newton-Raphson method and has quadratic convergence.

Now, we extend this idea to finding the unknown Lagrange multiplier. The main step is to first take the Laplace transform to Eq. (1). Then the linear part is transformed into an algebraic equation as follows:

$$
s^{m} U(s)-u^{(m-1)}(0)-\cdots-s^{m-1} u(0)+L[R[u]]+L[N[u]]-L[g(t)]=0,
$$

where $U(s)=L[u(t)]=\int_{0}^{\infty} e^{-s t} u(t) d t$.

The iteration formula of (7) can be used to suggest the main iterative scheme involving the Lagrange multiplier as

$$
\begin{aligned}
U_{n+1}(s)= & U_{n}(s)+\lambda(s)\left[s^{m} U_{n}(s)-u^{(m-1)}(0)-\cdots-s^{m-1} u(0)\right. \\
& \left.+L\left(R\left[u_{n}\right]+N\left[u_{n}\right]-g(t)\right)\right] .
\end{aligned}
$$

Considering $L\left(R\left[u_{n}\right]+N\left[u_{n}\right]\right)$ as restricted terms, one can derive a Lagrange multiplier as

$$
\lambda(s)=-\frac{1}{s^{m}}
$$

With Eq. (9) and the inverse-Laplace transform $L^{-1}$, the iteration formula (8) can be explicitly given as

$$
\begin{aligned}
u_{n+1}(t)= & u_{n}(t)-L^{-1}\left[\frac { 1 } { s ^ { m } } \left[s^{m} U_{n}(s)-u^{(m-1)}(0)+\cdots-s^{m-1} u(0)\right.\right. \\
& \left.\left.+L\left(R\left[u_{n}\right]+N\left[u_{n}\right]-g(t)\right)\right]\right] \\
= & L^{-1}\left(\frac{1}{s^{m}} u^{(m-1)}(0)+\cdots+\frac{u(0)}{s}-\frac{1}{s^{m}} L\left(R\left[u_{n}\right]+N\left[u_{n}\right]-g(t)\right)\right),
\end{aligned}
$$

where the initial iteration $u_{0}(t)$ can be determined by

$$
u_{0}(t)=L^{-1}\left(\frac{1}{s^{m}} u^{(m-1)}(0)+\cdots+\frac{u(0)}{s}\right)=u(0)+u^{\prime}(0) t+\cdots+\frac{u^{(m-1)}(0) t^{m-1}}{(m-1) !} .
$$

Eq. (11) also explained why the initial iteration in the classical VIM is determined by the Taylor series.

This modified VIM here transfers the problem into the partial differential equation in the Laplace $s$-domain and removes the differentiation with respect to time. This idea has been used in other analytical methods such as the Laplace ADM $[23,24]$ and the Laplace HPM [25], respectively.

\section{Illustrative examples}

We now consider the applications of the modified VIM to both ODEs and FDEs. 


\subsection{Ordinary differential equations}

Example 1 Consider the following simple line differential equation:

$$
\frac{d u}{d t}+u=0, \quad u(0)=u_{0},
$$

which has the exact solution $u(t)=u_{0} e^{-t}$.

We can obtain the successive approximate solutions as

$$
\begin{aligned}
& u_{0}(t)=u(0)=u_{0}, \\
& u_{1}(t)=L^{-1}\left(\frac{1}{s}-\frac{1}{s^{2}}\right)=u_{0}(1-t), \\
& u_{2}(t)=L^{-1}\left(\frac{1}{s}-\frac{1}{s^{2}}+\frac{1}{s^{3}}\right)=u_{0}\left(1-t+\frac{t^{2}}{2}\right) \text {, } \\
& u_{n}(t)=L^{-1}\left(\frac{1}{s}-\frac{1}{s^{2}}+\cdots+(-1)^{n+1} \frac{1}{s^{n+1}}\right)=u_{0}\left[\sum_{k=0}^{n} \frac{(-t)^{n}}{n !}\right] \text {. }
\end{aligned}
$$

For $n \rightarrow \infty, u_{n}(t)$ tends to the exact solution $u_{0} e^{-t}$.

Example 2 The logistic differential equation [26]

$$
\frac{d u}{d t}=u(1-u), \quad u(0)=\frac{1}{2}
$$

has the exact solution $u(t)=\frac{e^{t}}{1+e^{t}}$. By the present VIM, we have the following solutions:

$$
\begin{aligned}
& u_{0}(t)=\frac{1}{2} \\
& u_{1}(t)=L^{-1}\left(\frac{1}{2 s}+\frac{1}{4 s^{2}}\right)=\frac{1}{2}+\frac{1}{4} t \\
& u_{2}(t)=L^{-1}\left(\frac{1}{2 s}+\frac{1}{4 s^{2}}-\frac{1}{8 s^{4}}\right)=\frac{1}{2}+\frac{1}{4} t-\frac{1}{48} t^{3},
\end{aligned}
$$

The same solutions using the classical VIM can be found in [26].

On the other hand, if we use $d u / d t$ and the linear term $u$ when determining the Lagrange multiplier, we can derive a Lagrange multiplier explicitly

$$
\delta U_{n+1}(s)=\delta U_{n}(s)+\delta \lambda\left[s U_{n}(s)-u(0)-U_{n}(s)\right]=\delta U_{n}(s)+\lambda(s)(s-1) \delta U_{n}(s)
$$

and

$$
\lambda(s)=-\frac{1}{s-1} .
$$


There can be various choices of $u_{0}(t)$ and $\lambda(s)$ which affect the speed of the convergence. We note that the integration by parts is not used and the calculation of the Lagrange multiplier here is much simpler. Furthermore, the VIM can be easily extended to FDEs and this is the main purpose of our work.

\subsection{Fractional differential equations}

In the early application of VIM [2] to FDEs, the term ${ }_{0}^{C} D_{t}^{\alpha} u$ is considered as a restricted variation, i.e.,

$$
\frac{d u}{d t}+{ }_{0}^{C} D_{t}^{\alpha} u=g(t, u), \quad 0<t, 0<\alpha<1
$$

and the variational iteration formula is given as

$$
u_{n+1}=u_{n}+\int_{0}^{t} \lambda(t, \tau)\left(\frac{d u_{n}}{d \tau}+{ }_{0}^{C} D_{\tau}^{\alpha} u-g(\tau, u)\right) d \tau
$$

where ${ }_{0}^{C} D_{t}^{\alpha}$ is the Caputo derivative [27] and $g\left(\tau, u_{n}\right)$ is a nonlinear term.

But for the following FDEs, the above popular applications of the VIM were not successful:

$$
\begin{aligned}
& { }_{0}^{C} D_{t}^{\alpha} u+R[u]+N[u]=g(t), \\
& u^{(k)}\left(0^{+}\right)=a_{k}, \quad 0<t, 0<\alpha, m=[\alpha]+1, k=0, \ldots, m-1 .
\end{aligned}
$$

Now, we consider the application of the modified VIM.

The following Laplace transform [27-29] of the term ${ }_{0}^{C} D_{t}^{\alpha} u$ holds:

$$
L\left[{ }_{0}^{C} D_{t}^{\alpha} u\right]=s^{\alpha} U(s)-\sum_{k=0}^{m-1} u^{(k)}\left(0^{+}\right) s^{\alpha-1-k}, \quad m-1<\alpha \leq m .
$$

Taking the above Laplace transform to both sides of (18), the iteration formula of Eq. (18) can be constructed as

$$
U_{n+1}(s)=U_{n}(s)+\lambda(s)\left[s^{\alpha} U_{n}(s)-\sum_{k=0}^{m-1} u^{(k)}(0) s^{\alpha-k-1}+L\left(R\left[u_{n}\right]+N\left[u_{n}\right]-g(t)\right)\right]
$$

As a result, after the identification of a Lagrange multiplier $\lambda=-\frac{1}{s^{\alpha}}$, one can derive

$$
\begin{aligned}
u_{n+1}(t) & =u_{n}(t)-L^{-1}\left[\frac{1}{s^{\alpha}}\left[s^{\alpha} U_{n}(s)-\sum_{k=0}^{m-1} u^{(k)}(0) s^{\alpha-k-1}+L\left(R\left[u_{n}\right]+N\left[u_{n}\right]-g(t)\right)\right]\right] \\
& =L^{-1}\left(\sum_{k=0}^{m-1} u^{(k)}(0) s^{-k-1}-\frac{1}{s^{\alpha}} L\left(R\left[u_{n}\right]+N\left[u_{n}\right]-g(t)\right)\right), \quad m-1<\alpha \leq m
\end{aligned}
$$

and

$$
u_{0}(t)=L^{-1}\left(\sum_{k=0}^{m-1} u^{(k)}(0) s^{-k-1}\right)=u(0)+u^{\prime}(0) t+\cdots+\frac{u^{(m-1)}(0) t^{m-1}}{(m-1) !}
$$


Let us apply the above VIM to solve FDEs of Caputo type.

Example 3 Consider the relaxation oscillator equation

$$
{ }_{0}^{C} D_{t} u+\omega^{\alpha} u=0, \quad u(0)=1, \quad u^{\prime}(0)=0, \quad t>0,0<\alpha<2, \omega>0,
$$

with the exact solution $E_{\alpha}\left((-\omega t)^{\alpha}\right)[30]$, where $E_{\alpha}\left((-\omega t)^{\alpha}\right)$ denotes the Mittag-Leffler function.

After taking the Laplace transform to both sides of Eq. (22), we get the following iteration formula:

$$
U_{n+1}(s)=U_{n}(s)+\lambda(s)\left[s^{\alpha} U_{n}(s)-u(0) s^{\alpha-1}-u^{\left({ }^{\prime}\right)}\left(0^{+}\right) s^{\alpha-2}+\omega^{\alpha} L\left[u_{n}\right]\right]
$$

Setting $L\left[u_{n}(t)\right]$ as a restricted variation, $\lambda(s)$ can be identified as

$$
\lambda(s)=-\frac{1}{s^{\alpha}} .
$$

The approximate solution of Eq. (22) can be given as

$$
\begin{aligned}
u_{n+1}(t) & =u_{n}(t)-L^{-1}\left[\frac{1}{s^{\alpha}}\left[s^{\alpha} U_{n}(s)-u(0) s^{\alpha-1}-u^{\left({ }^{\prime}\right)}\left(0^{+}\right) s^{\alpha-2}+\omega^{\alpha} L\left[u_{n}\right]\right]\right] \\
& =L^{-1}\left[\frac{1}{s^{\alpha}}\left(u(0) s^{\alpha-1}+u^{\left({ }^{\prime}\right)}\left(0^{+}\right) s^{\alpha-2}-\omega^{\alpha} L\left[u_{n}\right]\right)\right],
\end{aligned}
$$

which reads

$$
\begin{aligned}
& u_{0}(t)=1, \\
& u_{1}(t)=1-\frac{\omega^{\alpha} t^{\alpha}}{\Gamma(1+\alpha)}, \\
& u_{2}(t)=1-\frac{\omega^{\alpha} t^{\alpha}}{\Gamma(1+\alpha)}+\frac{\omega^{2 \alpha} t^{2 \alpha}}{\Gamma(1+2 \alpha)},
\end{aligned}
$$

$u_{n}(t)$ rapidly tends to the exact solution of Eq. (24) for $n \rightarrow \infty$.

Example 4 Consider the fourth example, the time-fractional diffusion equation

$$
{ }_{0}^{C} D_{t}^{\alpha} u=\frac{\partial^{2} u(x, t)}{\partial x^{2}}+\frac{\partial(x u(x, t))}{\partial x}, \quad 0<\alpha<1, \quad u(x, 0)=x^{2} .
$$

The VIM solution of the fractional semi-derivative equation was developed by Das [31]. Other methods applied to this equation are available in [32] and the monographs [33, 34] in the fractional calculus.

We can have the following iteration formula for Eq. (25):

$$
\left\{\begin{array}{l}
u_{n+1}(t)=L^{-1}\left(\frac{x^{2}}{s}+\frac{1}{s^{\alpha}} L\left(\frac{\partial^{2} u_{n}(x, t)}{\partial x^{2}}+\frac{\partial\left(x u_{n}(x, t)\right)}{\partial x}\right)\right) \\
u_{0}(t)=x^{2}
\end{array}\right.
$$

and $\lambda(s)=-\frac{1}{s^{\alpha}}$ is used as earlier. 
As a result, the successive approximation can be obtained as follows:

$$
\begin{aligned}
& u_{0}(t)=x^{2}, \\
& u_{1}(t)=L^{-1}\left(\frac{x^{2}}{s}+\frac{\left(2+3 x^{2}\right)}{s^{\alpha+1}}\right)=x^{2} t+\frac{\left(2+3 x^{2}\right) t^{\alpha}}{\Gamma(1+\alpha)}, \\
& u_{2}(t)=x^{2} t+\frac{\left(2+3 x^{2}\right) t^{\alpha}}{\Gamma(1+\alpha)}+\frac{\left(8+9 x^{2}\right) t^{2 \alpha}}{\Gamma(1+2 \alpha)}, \\
& u_{3}(t)=x^{2} t+\frac{\left(2+3 x^{2}\right) t^{\alpha}}{\Gamma(1+\alpha)}+\frac{\left(8+9 x^{2}\right) t^{2 \alpha}}{\Gamma(1+2 \alpha)}+\frac{\left(26+27 x^{2}\right) t^{3 \alpha}}{\Gamma(1+3 \alpha)},
\end{aligned}
$$

The exact solution can be given in a compact form

$$
u(x, t)=\lim _{n \rightarrow \infty} u_{n}(x, t)=\lim _{n \rightarrow \infty} \sum_{i=0}^{n} \frac{k^{i} t^{i \alpha}}{\Gamma(1+i \alpha)}=E_{\alpha}\left(k t^{\alpha}\right)
$$

where $k^{i}=x^{2}+\left(1+x^{2}\right)\left(3^{i}-1\right)$.

The method's efficiency for a nonlinear differential equation with variable coefficients is illustrated in [35]. For other applications of a new modified VIM to ODEs and FDEs, readers are also referred to [36-38].

\section{Remarks}

(a) The conceived modification of the VIM is a universal approach to both ODEs and FDEs. As a result, it becomes possible to design a 'universal' software package in future work.

(b) Now one can consider implementing other linearized techniques, i.e., the Adomian series and the homotopy series to handle the nonlinear terms and improve the accuracy of the approximate solutions.

(c) This modified VIM can also be used to solve the FDEs of RL type.

\section{Conclusions}

A new approach is proposed to identify the Lagrange multipliers of the VIM and a concept of the Laplace-Lagrange multipliers is proposed from the Laplace transform. Especially for the FDEs, to the best of our knowledge, there is no effective method to identify the Lagrange multipliers. With the approach given in this paper, we can easily derive Lagrange multipliers without tedious calculation and new variational iteration formulae can be derived. Some FDEs with the Caputo derivatives are illustrated. The results show the modified method's efficiency compared with other versions of the VIM in fractional calculus. 


\section{Author details}

${ }^{1}$ College of Mathematics and Information Science, Neijiang Normal University, Neijiang, 641112 , China. ${ }^{2}$ College of Water Resources and Hydropower, Sichuan University, Chengdu, Sichuan 610065, China. ${ }^{3}$ Department of Mathematics and Computer Sciences, Cankaya University, Balgat, Ankara 06530, Turkey. ${ }^{4}$ Institute of Space Sciences, Magurele, Bucharest, Romania. ${ }^{5}$ Department of Mathematics, King Abdulaziz University, Jeddah, Saudi Arabia.

\section{Acknowledgements}

The authors would like to express their deep gratitude to the referees for their valuable suggestions and comments. The work is financially supported by the NSFC (11061028) and the key program of the NSFC (51134018).

\section{Received: 19 October 2012 Accepted: 6 January 2013 Published: 21 January 2013}

\section{References}

1. Inokuti, M, Sekine, H, Mura, T: General use of the Lagrange multiplier in nonlinear mathematical physics. In: Nemat-Nasser, S (ed.) Variational Methods in the Mechanics of Solids, pp. 156-162. Pregman Press, New York (1978)

2. He, JH: Approximate analytical solution for seepage flow with fractional derivatives in porous media. Comput. Methods Appl. Mech. Eng. 167(1-2), 57-68 (1998)

3. $\mathrm{He}, \mathrm{JH}$ : Variational iteration method - a kind of non-linear analytical technique: some examples. Int. J. Non-Linear Mech. 34(4), 699-708 (1999)

4. $\mathrm{He}, \mathrm{JH}$ : An elementary introduction to recently developed asymptotic methods and nanomechanics in textile engineering. Int. J. Mod. Phys. B 22(21), 3487-3578 (2008)

5. Abbasbandy, S: A new application of He's variational iteration method for quadratic Riccati differential equation by using Adomian's polynomials. J. Comput. Appl. Math. 207(1), 59-63 (2007)

6. Noor, MA, Mohyud-Din, ST: Variational iteration technique for solving higher order boundary value problems. Appl. Math. Comput. 189(2), 1929-1942 (2007)

7. Noor, MA, Mohyud-Din, ST: Variational iteration method for solving higher-order nonlinear boundary value problems using He's polynomials. Int. J. Nonlinear Sci. Numer. Simul. 9(2), 141-156 (2008)

8. Yusufoglu, E: The variational iteration method for studying the Klein-Gordon equation. Appl. Math. Lett. 21(7), 669-674 (2008)

9. Yıldırım, A, Öziş, T: Solutions of singular IVPs of Lane-Emden type by the variational iteration method. Nonlinear Anal., Theory Methods Appl. 70(6), 2480-2484 (2009)

10. Wu, GC: New trends in variational iteration method. Commun. Fract. Calc. 2(2), 59-75 (2011)

11. Wu, GC, Wu, KT: Variational approach for fractional diffusion-wave equations on Cantor sets. Chin. Phys. Lett. 29(6), Article ID 060505 (2012)

12. Wu, GC: Variational iteration method for q-difference equations of second order. J. Appl. Math. 2012, Article ID $102850(2012)$

13. Allahviranloo, T, Abbasbandy, S, Rouhparvar, H: The exact solutions of fuzzy wave-like equations with variable coefficients by a variational iteration method. Appl. Soft Comput. 11(2), 2186-2192 (2011)

14. Jafari, $\mathrm{H}$, Khalique, $\mathrm{CM}$ : Homotopy perturbation and variational iteration methods for solving fuzzy differential equations. Commun. Fract. Calc. 3(1), 38-48 (2012)

15. Jafari, $H$, Saeidy, M, Baleanu, D: The variational iteration method for solving $n$-th order fuzzy differential equations Cent. Eur. J. Phys. 10(1), 76-85 (2012)

16. $\mathrm{He}, \mathrm{JH}, \mathrm{Wu}, \mathrm{XH}$ : Variational iteration method: new development and applications. Comput. Math. Appl. 54(7-8), 881-894 (2007)

17. Shawagfeh, NT: Analytical approximate solutions for nonlinear fractional differential equations. Appl. Math. Comput. $131(2-3), 517-529(2002)$

18. Ray, SS, Bera, RK: An approximate solution of a nonlinear fractional differential equation by Adomian decomposition method. Appl. Math. Comput. 167(1), 561-571 (2005)

19. Duan, JS, Rach, R, Baleanu, D, Wazwaz, AM: A review of the Adomian decomposition method and its applications to fractional differential equations. Commun. Fract. Calc. 3(2), 73-99 (2012)

20. Wang, Q: Homotopy perturbation method for fractional KdV-Burgers equation. Chaos Solitons Fractals 35(5), 843-850 (2008)

21. Momani, S, Odibat, Z: Homotopy perturbation method for nonlinear partial differential equations of fractional order. Phys. Lett. A 365(5-6), 345-350 (2007)

22. Kadem, A, Baleanu, D: Homotopy perturbation method for the coupled fractional Lotka-Volterra equations. Rom. J. Phys. 56(3-4), 332-338 (2011)

23. Tsai, PY, Chen, CK: An approximate analytic solution of the nonlinear Riccati differential equation. J. Franklin Inst. 347(10), 1850-1862 (2010)

24. Zeng, DQ, Qin, YM: The Laplace-Adomian-Pade technique for the seepage flows with the Riemann-Liouville derivatives. Commun. Fract. Calc. 3(1), 26-29 (2012)

25. Javidi, M, Raji, MA: Combination of Laplace transform and homotopy perturbation method to solve the parabolic partial differential equations. Commun. Fract. Calc. 3(1), 10-19 (2012)

26. Wazwaz, AM: The variational iteration method for analytic treatment for linear and nonlinear ODEs. Appl. Math Comput. 212(1), 120-134 (2009)

27. Oldham, KB, Spanier, J: The Fractional Calculus. Academic Press, New York (1974)

28. Podlubny, I: Fractional Differential Equations. Academic Press, San Diego (1999)

29. Kilbas, AA, Srivastav, HM, Trujillo, JJ: Theory and Applications of Fractional Differential Equations. Elsevier, New York (2006)

30. Mainardi, F: Fractional relaxation-oscillation and fractional diffusion-wave phenomena. Chaos Solitons Fractals 7(9), 1461-1477 (1996)

31. Das, S: Analytical solution of a fractional diffusion equation by variational iteration method. Comput. Math. Appl. 57(3), 483-487 (2009) 
32. Hristov, J: Heat-balance integral to fractional (half-time) heat diffusion sub-model. Therm. Sci. 14(2), 291-316 (2010)

33. Diethelm, K: The Analysis of Fractional Differential Equations. Springer, Berlin (2010)

34. Baleanu, D, Diethelm, K, Scalas, E, Trujillo, JJ: Fractional Calculus Models and Numerical Methods. World Scientific, Singapore (2012)

35. Wu, GC: Challenge in the variational iteration method - a new approach to the identification of the Lagrange multipliers. J. King Saud Univ., Sci. (2012, in press). doi:10.1016/i.jksus.2012.12.002

36. Hristov, J: An exercise with the He's variation iteration method to a fractional Bernoulli equation arising in transient conduction with non-linear heat flux at the boundary. Int. Rev. Chem. Eng. 4(5), 489-497 (2012)

37. Wu, GC, Baleanu, D: Variational iteration method for the Burgers' flow with fractional derivatives-new Lagrange multipliers. Appl. Math. Model. (2012, in press). doi:10.1016/j.apm.2012.12.018

38. Wu, GC: Variational iteration method for solving the time-fractional diffusion equations in porous medium. Chin. Phys. B 21(12), Article ID 120504 (2012)

doi:10.1186/1687-1847-2013-18

Cite this article as: Wu and Baleanu: Variational iteration method for fractional calculus - a universal approach by Laplace transform. Advances in Difference Equations 2013 2013:18.

\section{Submit your manuscript to a SpringerOpen ${ }^{\odot}$ journal and benefit from:}

- Convenient online submission

- Rigorous peer review

- Immediate publication on acceptance

- Open access: articles freely available online

- High visibility within the field

- Retaining the copyright to your article 